\section{THU0057B TNF-ALPHA INDUCES NECROPTOSIS-LIKE DEATH OF MACROPHAGES AND PROMOTES EXTRACELLULAR RELEASE OF 14-3-3ETA}

Gulzhan Trimova ${ }^{1}$, Kaoru Yamagata ${ }^{1}$, Shigeru Iwata ${ }^{1}$, Tong Zhang ${ }^{1}$, Fumi Uemura', Minoru Satoh ${ }^{2}$, Michelle Zaharik ${ }^{3}$, Norma Biln ${ }^{3}$, Shintaro Hirata ${ }^{4}$ Shingo Nakayamada ${ }^{1}$, Yoshiya Tanaka ${ }^{1} .{ }^{1}$ University of Occupational and Environmental Health, Japan, The First Department of Internal Medicine, Kitakyushu, Japan; ${ }^{2}$ University of Occupational and Environmental Health, Japan, Department of Clinical Nursing, School of Health Sciences, Kitakyushu, Japan; ${ }^{3}$ Augurex Life Sciences Corp, North Vancouver, BC, Canada; ${ }^{4}$ Hiroshima University Hospital, Hiroshima, Japan

Background: $14-3-3 \eta$ is an intracellular protein detected in the serum and synovial fluid of patients with rheumatoid arthritis (RA) [1]. While presence of 14-3-3 $\eta$ is both diagnostic for early and established RA [2] and prognostic for radiographic progression [3], the mechanism of 14-3$3 \eta$ externalization in RA remains unclear.

Objectives: To clarify the mechanism of externalization of 14-3-3n into the extracellular space using human PBMC-derived macrophages (Mф). Methods: Distribution of $14-3-3 \eta$ in synovial tissue of patients with RA or osteoarthritis (OA) was examined by immunohistochemistry; cellular morphology was studied by confocal microscopy and electron microscopy (EM). M $\phi$ were stimulated with TNF- $\alpha$, Diamide (induces ligand-independent TNFR signaling) or IL-6/sIL-6R. Western blotting was used to detect S-358-phosphorylated MLKL (a mediator of necroptosis) and presence of 14-3-3 $\eta$ in $M \phi$ culture supernatants.

Results: Dense and widespread staining of $14-3-3 \eta$ was detected in $M \phi$ of RA, but not OA, synovial tissues. 14-3-3 $\eta$ and peptidylarginine deiminase 4 (PAD4) co-localized in $\mathrm{CD}^{+} 8^{+}$cells $(\mathrm{M} \phi)$ from RA synovial tissue; 14-3-3 $\eta$ was not detected in $\mathrm{CD}^{+} 8^{+}$cells from RA lung tissue or $\mathrm{CD}^{+}$ cells ( $T$ cells) from RA synovium. The outer space around the nucleus of healthy control $M \phi$ treated with $T N F-\alpha$ or Diamide, but not IL-6/sIL-6R, demonstrated abnormal actin distribution by phalloidin staining and presence of cellular and organelle swelling by EM. Further, magnified images showed partial destruction of the cell membrane in TNF- $\alpha$ and Diamidetreated cells. Phosphorylation of MLKL was observed between $20 \mathrm{~min}$ and $24 \mathrm{~h}$ after stimulation of healthy control $M \phi$ with TNF- $\alpha$, with maximum signal at $8 \mathrm{~h}$ post-stimulation, but was not observed upon IL-6/sIL$6 \mathrm{R}$ stimulation at any time point. After $8 \mathrm{~h}$ of stimulation no 14-3-3 $\eta$ was detected in the culture supernatant of healthy control $M \phi$ endogenously expressing $14-3-3 \eta$ or $M \phi$ stimulated with IL-6/sIL-6R, while a high concentration of $14-3-3 \eta$ was detected in culture supernatants in $M \phi$ treated with TNF- $\alpha$ or Diamide.

Conclusion: $14-3-3 \eta$ protein was abundant in RA, but not OA, synovial tissues and co-localized with PAD4 in $\mathrm{CD}^{+} 8^{+}$synovial $\mathrm{M} \phi$; this close proximity to PAD4 may promote citrullination of $14-3-3 \eta$ in vivo. Treatment of healthy control $M \phi$ with TNF- $\alpha$ induced phosphorylation of MLKL and cell swelling and disintegration of the plasma membrane characteristic of nec-

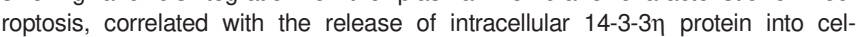
lular supernatants. Our results shed light on mechanism of externalization of $14-3-3 \eta$ and how it achieves elevated levels in RA synovial fluid.

\section{REFERENCES:}

[1] Kilani RT, et al. J Rheumatol 2007; 34:1650-7.

[2] Maksymowych W, et al. J Rheumatol 2014; 41:2104-13.

[3] Carrier N, et al. Arthritis Res Ther 2016; 18:50.

Competing Interests: M. Zaharik and N. Biln are employees of Augurex $\mathrm{Y}$. Tanaka, has received speaking fees and/or honoraria from Daiichi-Sankyo, Astellas, Eli Lilly, Chugai, Sanofi, Abbvie, Pfizer, YL Biologics, Bristol-Myers, Glaxo-Smithkline, UCB, Mitsubishi-Tanabe, Novartis, Eisai, Takeda, Janssen, Asahi-kasei and has received research grants from Mitsubishi-Tanabe, Bristol-Myers, Eisai, Chugai, Takeda, Abbvie, Astellas, Daiichi-Sankyo, Ono, MSD, Taisho-Toyama. All other authors have nothing to disclose.

Disclosure of Interests: Gulzhan Trimova: None declared, Kaoru Yamagata: None declared, Shigeru Iwata: None declared, Tong Zhang: None declared, Fumi Uemura: None declared, Minoru Satoh: None declared, Michelle Zaharik: None declared, Norma Biln Shareholder of: Amgen, Employee of: Pfizer, Amgen and Abbott, Shintaro Hirata Grant/research support from: Eli Lilly, UCB, Consultant for: Bristol-Myers Squibb, Jansen, UCB, Paid instructor for: AbbVie, Eisai, Tanabe-Mitsubishi, Speakers bureau: AbbVie, Astellas, Ayumi, Bristol-Myers Squibb, Chugai, Eisai, Eli Lilly, Jansen, Kissei, Pfizer, Sanofi, Takeda, Tanabe-Mitsubishi, UCB, Shingo Nakayamada Grant/research support from: Mitsubishi-Tanabe, Takeda, Novartis and MSD, Speakers bureau: Bristol-Myers, Sanofi, Abbvie, Eisai, Eli Lilly, Chugai, Asahi-kasei and Pfizer, Yoshiya Tanaka Grant/research support from: Abbvie, Astellas, Bristol-Myers Squibb,
Chugai, Daiichi-Sankyo, Eisai, Mitsubishi-Tanabe, MSD, Ono, TaishoToyama, Takeda, Speakers bureau: Abbvie, Asahi-kasei, Astellas, BristolMyers Squibb, Chugai, Daiichi-Sankyo, Eli Lilly, Eisai, Glaxo-Smithkline Janssen, Mitsubishi-Tanabe, Novartis, Pfizer Japan Inc, Sanofi, Takeda, UCB, YL Biologics

DOI: 10.1136/annrheumdis-2019-eular.3886

\section{Rheumatoid arthritis - prognosis, predictors and outcome}

\section{THU0058 B CELL SYNOVITIS AND CLINICAL PHENOTYPES IN RHEUMATOID ARTHRITIS AT DIFFERENT DISEASE STAGES}

Felice Rivellese ${ }^{1}$, Frances Humby ${ }^{1}$, Serena Bugatti ${ }^{2}$, Liliane Fossati-Jimack ${ }^{1}$, Hasan Rizvi ${ }^{3}$, Davide Lucchesi ${ }^{1}$, Gloria Lliso Ribera ${ }^{1}$, Alessandra Nerviani ${ }^{1}$, Rebecca Hands ${ }^{1}$, Giovanni Giorli ${ }^{1}$, Barbara Frias ${ }^{1}$, Edyta Jaworska ${ }^{1}$,

Katriona Goldmann ${ }^{1}$, Myles Lewis ${ }^{1}$, Antonio Manzo ${ }^{2}$, Michele Bombardieri ${ }^{1}$, Costantino Pitzalis'. ${ }^{1}{ }^{1}$ Queen Mary University of London, Centre for Experimental Medicine and Rheumatology, London, United Kingdom; ${ }^{2}$ University of Pavia, IRCCS Policlinico San Matteo Foundation, Pavia, Italy; ${ }^{3}$ Barts Health NHS Trust, Department of Pathology, London, United Kingdom

Background: The role of $B$ cells in the pathogenesis of Rheumatoid Arthritis (RA) is well recognised and has been reinforced by the established efficacy of B cell depleting treatments. However, B cell infiltration in synovia is highly variable and their association with clinical disease activity has been inconsistently reported, with contradicting results possibly linked to the lack of standardization in quantitative and qualitative assessment of B cell synovitis. In particular, the presence of B cells in synovia has never been systematically assessed in large cohorts.

Objectives: To evaluate B cells and their association with clinical phenotypes in the synovia of patients with RA at various disease stages.

Methods: A total of 432 synovial biopsies from the following cohorts of RA patients were analysed: i. early $(<1$ year $)$ treatment-naïve RA $(n=165)$, ii. Synthetic Disease Modifying Anti-Rheumatic Drugs inadequate responders (sDMARDs-ir) $(n=103)$, iii. TNF-inhibitors inadequate responders (TNFi-ir) ( $n=164)$. Haematoxylin and eosin staining was used for the assessment of synovitis according to a previously validated score (Krenn). Upon immunohistochemical staining for CD20, semi-quantitative (Sq) scoring (0-4) was used to classify patients into B cell rich $(\geq 2)$ and poor $(<2)$ and automated digital image analysis (DIA) to calculate the B cell area fraction. B cell expression markers, including CD20 mRNA counts and a composite $B$ cell module, were obtained by RNA-sequencing from early RA synovial biopsies $(n=91)$.

Results: Semi-quantitative synovial B cell scores positively correlated with the B cell area fraction obtained by DIA (Spearman $r 0.93$ in early RA and 0.88 in TNFi-ir, $p<0.0001$ ). Accordingly, B cell rich patients (Sq score $\geq 2$ ) had a significantly higher $B$ cell area fraction $(p<0.0001)$. RNAsequencing from 91 patients with early RA showed a positive correlation between the $\mathrm{Sq} B$ cell scores and CD20 mRNA counts and the B cell module (Spearman $r=0.6$ and 0.67 , respectively, $p<0.0001$ ). Similarly, a positive correlation was found between the $\mathrm{B}$ cell area fraction obtained by DIA and CD20 mRNA counts and B cell module ( $r=0.67$ and 0.69 , respectively, $p<0.0001$ ) When comparing $B$ cell presence in the three cohorts, B cell-rich synovitis was present in $35 \%$ of early RA, $36 \%$ of sDMARDs-ir and $47.1 \%$ of TNFi-ir $(p=0.025$ comparing early RA vs latestage TNFi-ir patients). Finally, while $B$ cell-rich patients showed significantly higher synovial inflammatory scores across all cohorts, higher disease activity (number of swollen joints, DAS28) and higher prevalence of autoantibody positivity (ACPA and RF) in B cell-rich patients were observed exclusively in the early RA cohort.

Conclusion: We here describe a robust and validated synovial B cell score that can potentially contribute to patient stratification in RA, as it helps identifying an enrichment of $B$ cell synovitis in established disease, uncoupled from clinical disease activity.

Acknowledgement: We would like to thank all the investigators and recruitment centers from PEAC (http://www.peac-mrc.mds.qmul.ac.uk centres.php), STRAP (http://www.matura-mrc.whri.qmul.ac.uk/strap_recruiting_centers.php) and R4RA (http://www.rura-nihr.whri.qmul.ac.uk/recruiting_centres.php) and the EMR clinical trial team at Queen Mary (http://www. r4ra-nihr.whri.qmul.ac.uk/docs/contributors_r4ra-for_website.pdf)

Disclosure of Interests: Felice Rivellese: None declared, Frances Humby: None declared, Serena Bugatti Speakers bureau: Bristol-Myers Squibb, Celgene, Lilly, Novartis, Sanofi, Janssen, Liliane Fossati-Jimack: None declared, Hasan Rizvi: None declared, Davide Lucchesi: None declared, 